\title{
Hysteresis Nonlinearity Identification Using New Preisach Model-Based Artificial Neural Network Approach
}

\author{
Mohammad Reza Zakerzadeh, ${ }^{1}$ Mohsen Firouzi, ${ }^{2}$ \\ Hassan Sayyaadi, ${ }^{1}$ and Saeed Bagheri Shouraki ${ }^{2}$ \\ ${ }^{1}$ School of Mechanical Engineering, Sharif University of Technology, P.O. Box 11155-9567, Tehran, Iran \\ ${ }^{2}$ Electrical Engineering Department, Artificial Creature Lab, Sharif University of Technology, \\ P.O. Box 11155-9567, Tehran, Iran \\ Correspondence should be addressed to Mohammad Reza Zakerzadeh, \\ mzakerzadeh@mech.sharif.edu
}

Received 29 September 2010; Revised 26 December 2010; Accepted 15 February 2011

Academic Editor: I. Stamova

Copyright (c) 2011 Mohammad Reza Zakerzadeh et al. This is an open access article distributed under the Creative Commons Attribution License, which permits unrestricted use, distribution, and reproduction in any medium, provided the original work is properly cited.

\begin{abstract}
Preisach model is a well-known hysteresis identification method in which the hysteresis is modeled by linear combination of hysteresis operators. Although Preisach model describes the main features of system with hysteresis behavior, due to its rigorous numerical nature, it is not convenient to use in real-time control applications. Here a novel neural network approach based on the Preisach model is addressed, provides accurate hysteresis nonlinearity modeling in comparison with the classical Preisach model and can be used for many applications such as hysteresis nonlinearity control and identification in SMA and Piezo actuators and performance evaluation in some physical systems such as magnetic materials. To evaluate the proposed approach, an experimental apparatus consisting one-dimensional flexible aluminum beam actuated with an SMA wire is used. It is shown that the proposed ANN-based Preisach model can identify hysteresis nonlinearity more accurately than the classical one. It also has powerful ability to precisely predict the higher-order hysteresis minor loops behavior even though only the first-order reversal data are in use. It is also shown that to get the same precise results in the classical Preisach model, many more data should be used, and this directly increases the experimental cost.
\end{abstract}

\section{Introduction}

Today hysteresis modeling is one of the most interesting and challenging field of study in many engineering applications such as shape memory alloy (SMA), piezoelectric, piezocermaic, magnetostrictive, and electromechanical actuators. Since unmodeled hysteresis causes inaccuracy in trajectory tracking and decreases the performance of control systems, 
an accurate modeling of hysteresis behavior for performance evaluation and identification as well as controller design is essentially needed. To overcome this drawback, it is necessary to develop hysteresis models that not only their parameters can easily and precisely be identified but also are suitable for real-time control and compensation system design [1].

Two different methods of modeling have been proposed to capture the observed hysteretic characteristics [2]. The first group of models is derived from the underlying physics of hysteresis and combined with empirical factors to describe the observed characteristics [3-5]. However, these models have limited applicability, as the physical basis of some of the hysteresis characteristics is not completely understood [6]. Furthermore, considerable effort is required in identifying and tuning the model parameters to accurately describe the hysteresis nonlinearity. Another major drawback of these physical models is that they are specific to a particular type of system, and this implies separate controller design techniques for each system [7].

The second group of models is based on the phenomenological nature and mathematically describes the observed phenomenon without necessarily providing physical insight into the problems [8-14]. Among these models, the Preisach model has found extensive application for modeling hysteresis in SMAs and other smart actuators [8, 10,14]. Although the Preisach model does not provide physical insight into the problem, it provides a means of developing phenomenological model that is capable of predicting behaviors similar to those of the physical systems. Therefore, it is a convenient tool for hysteresis identification and compensation [13].

In Preisach modeling technique overall system with hysteresis behavior is modeled by weighted parallel connections of nonideal relays termed as Preisach elemental operators, $\gamma_{\alpha, \beta}$ (Figures 1 and 2). Every elemental operator with output +1 or -1 (zero in some models) as a nonlinear operator consists of two parameters, $\alpha, \beta$, which denote upper and lower switching values of input, respectively. Along with the set of operators $\gamma_{\alpha, \beta}$ is an arbitrary weight function $\mu(\alpha, \beta)$, called the Preisach density function (PDF), which works as a local influence of each operator in overall hysteresis model.

There are two general approaches to implement the Preisach model. The first approach is trying to approximate the PDF by some predefined special forms with a few undetermined parameters. Each material has an optimal distribution function and parameter, which deliver the best result with respect to the experimental data [15]. In the identification process of this approach, the unknown parameters are determined in order to minimize the error between the output of the model and experimental data by numerical curve fitting algorithms such as minimum least square method. In other words, optimal fitting of the calculated outputs to measured data of the real system can determine the values of parameters used in the assumed function.

The main disadvantage of this approach is the fact that the accuracy of the model is strongly dependent on the type of the candidate function and the number of its parameters. Moreover, it is not easy to determine the suitable shape of the distribution functions by less experimental data. Also, to simulate the hysteresis loops of different materials, the different distribution functions and parameters are needed. In order to overcome this drawback, a numerical density function approximation method based on mapping Preisach model into a linear equation system was proposed by Shirley and Venkatraman [16]. Another solution is identifying the Preisach function by using artificial neural networks (ANNs) or fuzzy approximators. As a matter of fact, the neural networks or fuzzy engines provide the parameters necessary for describing a given hysteresis loop, under the assumption that the type of the Preisach function is already known. In [17], the identification of the Preisach 


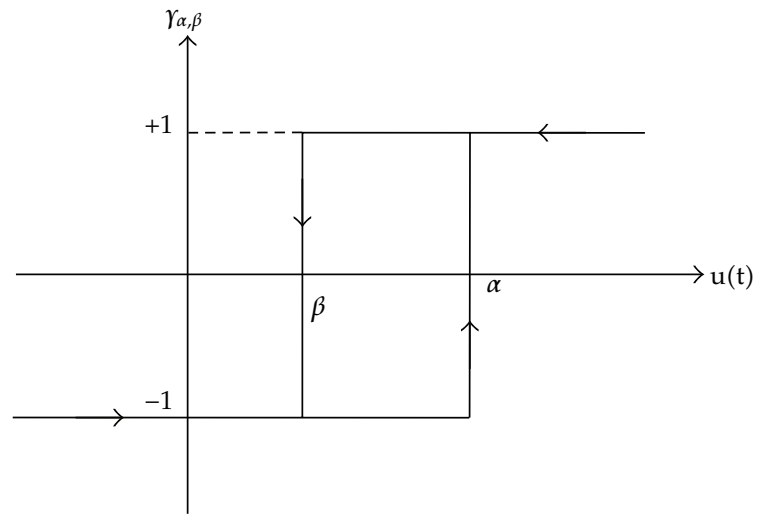

Figure 1: Preisach elemental operator.

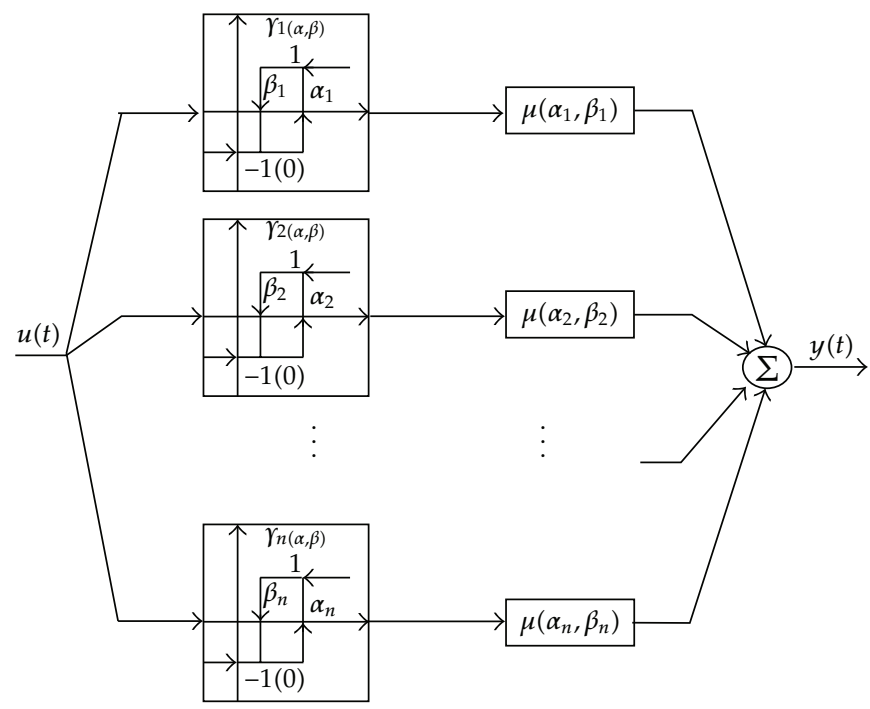

Figure 2: Block representation of the classical Preisach model.

function of a material is performed by using a neural network trained by a collection of hysteresis curves, whose Preisach functions are known. When a new hysteresis curve is given as input to this neural network, it is able to give as output both the functional dependence of the Preisach function and its numerical parameters. Moreover, the proposed method allows to determine any usual analytical structure of the Preisach function provided that a suitable training set is adopted. The method has been applied to the prediction of hysteresis loops of magnetic sheets by using Preisach method and it has shown a good numerical accuracy. In [18], two different identification techniques have been proposed. In this paper, hysteresis is modeled by applying the classical Preisach model whose identification procedure is performed by the adoption of both a fuzzy approximator and a feed-forward neural network to analytically reconstruct the Preisach distribution function, without any special smoothing of the measured data, owing to the filtering capabilities of the neurofuzzy interpolators. 
Since, in the first form of Preisach model, the numerical evaluation of double integral is a time consuming process that hinders the practical application of the Preisach model, in the second approach, a numerical evaluation of double integral instead of density function approximation developed by Mayergoyz was proposed [14]. Despite that the Mayergoyz approach has received general acceptance to capture the main features of hysteresis phenomena, it is not suitable for real-time control applications. There have been some limitations on the accuracy of this method in addition to its computation time for the model inversion. These are caused by the restrictions of switching points in the Preisach plane, the inaccuracy of data measurement, and the geometrical interpolation error appeared in the identification process of classical Preisach model [13].

Several extensions and modifications of the numerical classical Preisach model using fuzzy inference engine, artificial neural networks as well as neurofuzzy identifier have been presented in order to remedy these problems $[6,19,20]$. However, it is known that these tools can only be available for the approximation of the continuous systems with one-to one and multi-to-one mappings and they are unable to directly model the systems with multi-valued mapping such as hysteresis [21]. Therefore, they are needed to utilize the local memory property of the classical Preisach model.

Ahn and Kha [6] presented a new modification of the numerical classical Preisach based on geometrical implementation model using a fuzzy inference engine. The experimental evaluation showed that the model is suitable in predicting the hysteresis phenomenon of the SMA actuators. They also used the fuzziness based inverse Preisach model incorporated in a closed loop internal model control to investigate the control performance and the effect of hysteresis compensation for SMA actuators. In spite of strong power modeling of fuzzy inference engines (FIE) through a simple linguistic computation paradigm, these tools suffer from a lack of learning algorithms to adjust the best membership functions of input domains. But, on the other hand, the artificial neural networks have a good ability to map input-output patterns through straightforward learning algorithms. To overcome the aforementioned problems of FIEs and utilizing the excellent learning capability of neural networks, neurofuzzy tools are developed in the field of computational intelligent [22]. Dlala and Arkkio [19] proposed a method that identified the numerical classical Preisach model by using a neurofuzzy approximator instead of interpolation which is used in the Mayergoyz approach. This novel method utilizes the available data in the major loop in the identification process and omits the need of measuring the first-order reversal curves. They also applied their method to predict cyclic minor loops of a soft magnetic composite and verified the accuracy of proposed method with respect to experimental data. Since the adaptive learning process of neurofuzzy approach is much time, consuming, there is a limitation on the number of fuzzy rules. Therefore, often there is a trade-off between the system accuracy, computation run time and fuzzy rules number [20].

ANNs have powerful fault tolerant computing ability which has been used to model a wide range of systems for mathematical models which either cannot be defined or are illdefined. Similarly, ANNs are well suited for systems that involve complex, multivariable processes. One of the advantages of using neural networks for hysteresis modeling is that their parameters can be updated online to track the change of the environment or operating condition. It is demonstrated in this paper that ANNs are individually capable of modeling hysteresis based on classical Preisach approaches without suffering the mentioned problems of fuzzy inference engines as well as neurofuzzy systems. To evaluate this approach in hysteresis modeling, a one-dimensional flexible aluminum beam, whose deflection is controlled by an SMA wire as an actuator, is used. Experimental results show the power 
of the proposed ANN based Preisach hysteresis model in comparison with classical Preisach model and mentioned Shirley et al. approach.

The paper is organized as follows. Section 2 is dedicated to the classical Preisach model and its geometrical interpretation. In this section an introduction of the classical Preisach model is presented. The Numerical Preisach model is discussed in more detail in Section 3 and its implementation method is discussed in Section 4. Then, in Section 5 a method developed by Shirley et al. for PDF approximation is presented. In Section 6 the structure of proposed novel ANN based Preisach model is described. The evaluation of the presented model is compared with experimental results in Section 7. Finally, the concluding comments are provided in Section 8.

\section{Classical Preisach}

Preisach model is a famous hysteresis identification technique, which is first introduced on the base of phenomenological analysis of ferromagnetic materials by German physicist F. Preisach almost 75 years ago [23]. The Russian mathematician, Krasnoselskii, in 1970 represented Preisach model into a pure formulized mathematical form in which hysteresis is modeled by linear combination of hysteresis operators [24]. Mathematical form of the classical Preisach model can be sketched by equation:

$$
f(t)=\iint_{\alpha \geq \beta} \mu(\alpha, \beta) \gamma_{\alpha, \beta}[u(t)] d \alpha d \beta,
$$

where $f(t)$ is the output of the model at state $t$ and $u(t)$ is the input at the same state, and $\gamma_{\alpha, \beta}$ denotes elementary hysteresis operator with $\alpha$ and $\beta(\alpha \geq \beta)$ parameters as upper and lower switching values, respectively (see Figure 1). Output of elemental operators would be only +1 or -1 (zero in some models). In $(2.1), \mu(\alpha, \beta)$ is density function value or Preisach function corresponding to $\alpha$ and $\beta$ which should be determined by use of some experimental data. This distributed weighting describes the relative contribution of each relay in overall hysteresis system (see Figure 2). Equation (2.1) can be represented in summation form of finite number of rectangular elemental Preisach operators $\gamma_{\alpha_{k}, \beta_{k}}$ as

$$
f(t)=\sum_{j=1}^{N} \sum_{i=1}^{N} \mu\left(\alpha_{i}, \beta_{j}\right) \gamma_{\alpha_{i} \beta_{j}}[u(t)],
$$

in which

$$
\begin{gathered}
\alpha_{i}=\beta_{i}=\alpha_{1}-2 \frac{(i-1)}{N-1} \alpha_{1}, \\
\gamma_{\alpha \beta}[u(t)]=\left\{\begin{array}{lc}
-1, & u(t) \leq \beta, \\
1, & u(t) \geq \alpha, \\
\text { maintain, } & \alpha \leq u(t) \leq \beta,
\end{array}\right.
\end{gathered}
$$




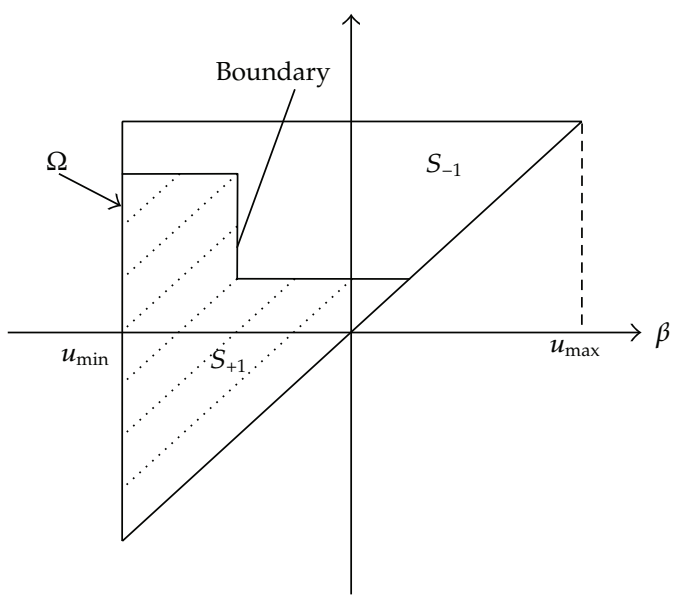

Figure 3: Geometrical representation of Preisach $(\alpha-\beta)$ plane.

where the approximation of dual integral form of classical Preisach model is represented by a dual sigma. Assume that normalized symmetrical hysteresis form $\alpha_{1}$ is up saturation point and $-\alpha_{1}$ is down saturation point.

For better representation of Preisach model, let us define a plane with $\alpha$ and $\beta$ coordinates where each elemental operator is denoted by a point $(\beta, \alpha)$ with a specific weighting value (Figure 3 ). PDF weighting values outside of Preisach triangle $(\Omega)$ must be zero, and also $u_{\max }$ and $u_{\min }$ denote upper and lower saturation points of input, respectively. When input is monotonically decreased or increased through $\alpha=\beta$ line in $\alpha-\beta$ plane, boundary line divides $\alpha-\beta$ plane to $S_{-1}$ and $S_{+1}$ area.

Dashed area in Figure 3 denoted by $S_{+1}$ consists of points $\left\{\alpha_{k}, \beta_{k} \mid \gamma_{\alpha_{k}, \beta_{k}}=+1\right\}$, and blank area denoted by $S_{-1}$ consists of points $\left\{\alpha_{k}, \beta_{k} \mid \gamma_{\alpha_{k}, \beta_{k}}=-1\right\}$; therefore (2.1) can be simplified to

$$
f(t)=\iint_{S_{+}} \mu(\alpha, \beta) d \alpha d \beta-\iint_{S_{-}} \mu(\alpha, \beta) d \alpha d \beta
$$

From the above description, the output of Preisach model depends on the subdivision of triangle $\Omega$. Equation (2.4) and its geometric representation is pure mathematical form of phenomenological Preisach model that was presented by Krasnoselskii. In order to implement the Preisach model for experimental applications, this primitive mathematical form of Preisach model was extended into a simple numerical form by Mayergoyz [25] that is discussed in the next section. It is worth mentioning that as an alternative method, if the form of density function in (2.4) (i.e., $\mu(\alpha, \beta)$ ) is predicted with respect to some experimental data, then the output of system can be easily calculated and this approach is described in Section 4.

\section{Numerical Preisach Model}

In addition to some numerical methods for Preisach density function approximation in hysteresis identification, there is a well-known and simpler numerical representation form of 
Preisach model in which output of hysteresis system is calculated by summation of specific terms. These terms depend on history of local maximum and minimum of system input by specific functionality. This method is discussed precisely in this section.

At first, assume that input diagram in Figure 4 is applied to the target hysteresis system. It is worth mentioning that since, in most applications of SMA actuators, the input is electrical current and the current does not get negative value, in these cases the Preisach plane is in the first quarter of coordinate system. Let input increase from 0 to $\alpha_{1}$ in time $t_{1}$, so all hysteresis operators with $\alpha_{i}<\alpha_{1}$ are on upper switching value and the others are on lower switching state. As it is discussed before, geometrically it results in subdivision of $\alpha-\beta$ plane triangle by line $u(t)=\alpha$ (see Figure 5). Upward motion through the line $u(t)=\alpha$ in Preisach plane is terminated when input reaches the local maximum value $\alpha_{1}$. By using (2.4), output $y_{\alpha_{1}}$ in this time can be expressed as

$$
y_{\alpha_{1}}=\iint_{S_{\alpha 1}^{+}} \mu(\alpha, \beta) \mathrm{d} \alpha \mathrm{d} \beta
$$

Then suppose that input is monotonically decreased to value $\beta_{1}$ in time $t_{2}$. As the input is being decreased through the line $u(t)=\beta$ in Preisach plane, all elemental operators in subdivision $S^{+}$with $\beta_{i}>u(t)$ are turned off; so their outputs become zero. It makes triangle $S^{+}$in Figure 5 divide into two regions (see Figure 6); therefore $S^{+}$triangle region in Figure 5 has been switched to a trapezoid in Figure 6. This right-to-left motion of $u(t)=\beta$ line is terminated when input reaches local minimum value $\beta_{1}$. At this time, output $y_{\alpha_{1} \beta_{1}}$ can be expressed as

$$
y_{\alpha_{1} \beta_{1}}=\iint_{S_{\alpha 1 \beta 1}^{+}} \mu(\alpha, \beta) \mathrm{d} \alpha \mathrm{d} \beta
$$

As a conclusion of this analysis, vertical and horizontal boundary lines between $\mathrm{S}^{+}$and $S^{0}$ regions in triangle $\alpha-\beta$ plane illustrate history of previous local maxima and minima of input, which affects current output value. This staircase boundary line is termed by memory interface $L(t)$. By generalizing the above discussion, at every instance of time, the output can be expressed as

$$
y(t)=\iint_{S^{+}(t)} \mu(\alpha, \beta) d \alpha d \beta
$$

If function $F(\alpha, \beta)$ is defined as

$$
F(\alpha, \beta)=y_{\alpha}-y_{\alpha \beta}
$$

it is equal to the output increments along the first-order transition curves. These curves are defined as follows: the input of the system hysteresis is monotonically increased from zero to some value $\alpha$ and then decreased to a value $\beta$, that is, greater than zero and smaller than $\alpha$. The term "first order" is used to emphasize the fact that each of these curves is formed after the first reversal of the input. It should be mentioned that the order of a minor loop branch is 


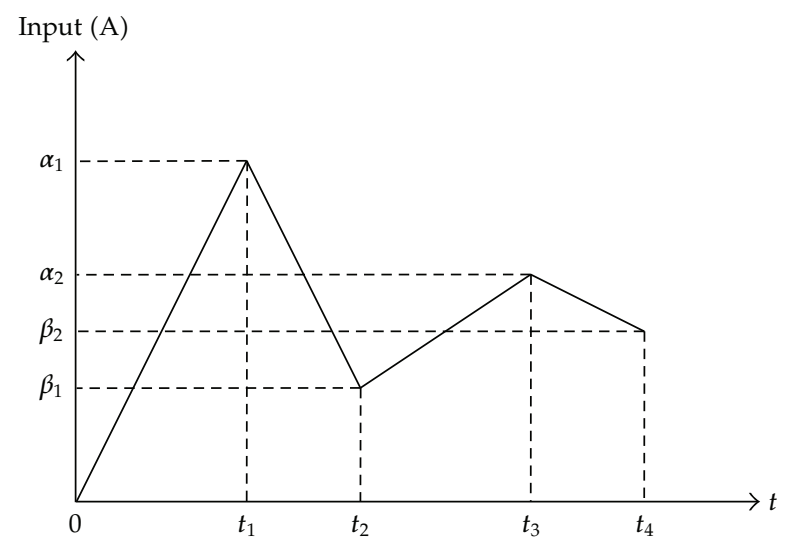

Figure 4: Input diagram.

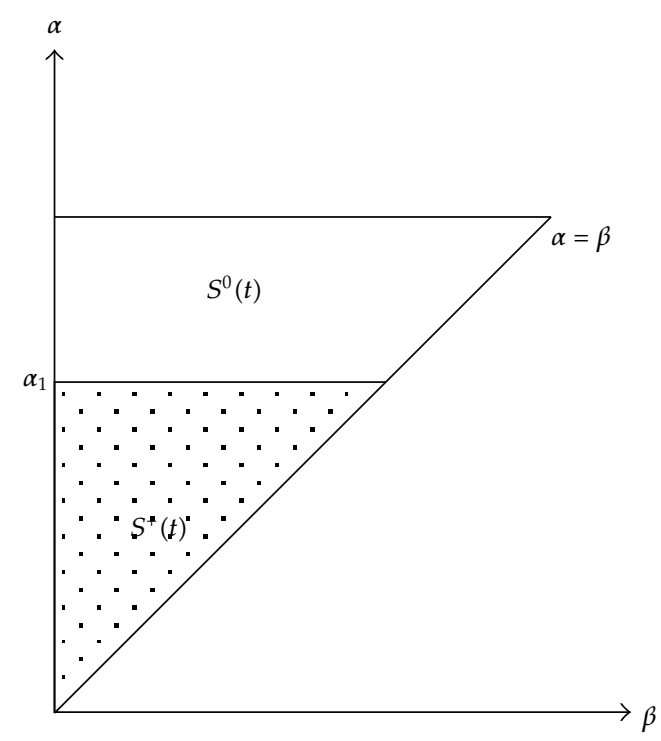

Figure 5: $\alpha$ - $\beta$ plane due to input increasing to $\alpha_{1}$.

defined to be the number of times that a hysteresis curve has reversed from a branch of the major hysteresis loop. Thus, all first-order branches are attached to one of the two branches of the major hysteresis loop. Similarly, the second-order branches are attached to the first-order branches, and the $n$ th-order branches are attached to the $n-1$ th-order branches [26].

From Figure 6 and (3.1), (3.2), (3.4),

$$
\iint_{T\left(\alpha_{1}, \beta_{1}\right)} \mu(\alpha, \beta) \mathrm{d} \alpha \mathrm{d} \beta=F\left(\alpha_{1}, \beta_{1}\right)
$$

where $T\left(\alpha_{1}, \beta_{1}\right)$ is a triangle which is shown in Figure 6. 


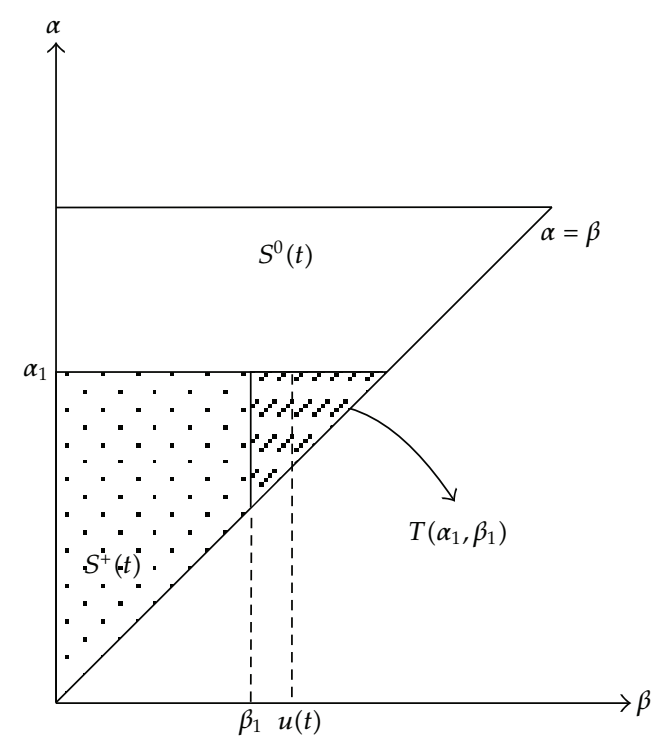

Figure 6: $\alpha-\beta$ plane due to input decreasing to $\beta_{1}$ from $\alpha_{1}$.

In the case that the input is monotonically increasing, $S^{+}(t)$ can be represented as trapezoidal regions plus one triangle region $\left(Q_{k}(t)\right.$ in Figures 7 and 8). Moreover, each trapezoidal region can be represented by two triangular region subtractions:

$$
\iint_{Q_{k}(t)} \mu(\alpha, \beta) \mathrm{d} \alpha \mathrm{d} \beta=\iint_{T\left(M_{k}, m_{k-1}\right)} \mu(\alpha, \beta) \mathrm{d} \alpha \mathrm{d} \beta-\iint_{T\left(M_{k}, m_{k}\right)} \mu(\alpha, \beta) \mathrm{d} \alpha \mathrm{d} \beta,
$$

where $M_{k}$ and $m_{k}$ denote maximum and minimum of input history. From (3.5) it is known that

$$
\begin{aligned}
\iint_{T\left(M_{k}, m_{k}\right)} \mu(\alpha, \beta) \mathrm{d} \alpha \mathrm{d} \beta & =F\left(M_{k}, m_{k}\right) \\
\iint_{T\left(M_{k}, m_{k-1}\right)} \mu(\alpha, \beta) \mathrm{d} \alpha \mathrm{d} \beta & =F\left(M_{k}, m_{k-1}\right) .
\end{aligned}
$$

Using (3.6)-(13), it is concluded that

$$
\iint_{Q_{k}(t)} \mu(\alpha, \beta) d \alpha d \beta=F\left(M_{k}, m_{k-1}\right)-F\left(M_{k}, m_{k}\right)
$$

Thus, in the case of decreasing input, as shown in Figure 8, the final link of boundary interface line $L(t)$ is vertical line $m_{n}=u(t)$ and output can be calculated as

$$
y(t)=\sum_{k=1}^{n(t)-1}\left[F\left(M_{k}, m_{k-1}\right)-F\left(M_{k}, m_{k}\right)\right]+F\left(M_{n}, m_{n-1}\right)-F\left(M_{n}, u(t)\right) .
$$




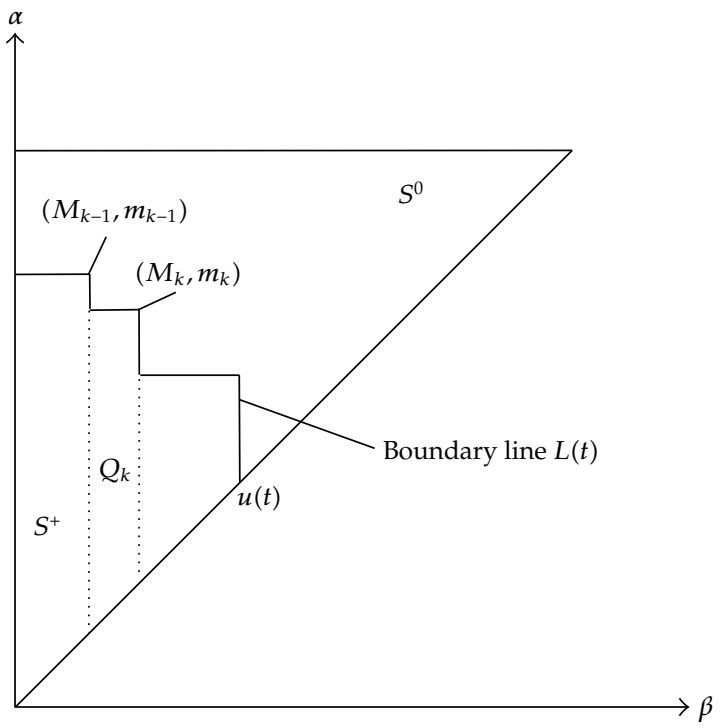

Figure 7: Numerical implementation of the Preisach model in the case of increasing input.

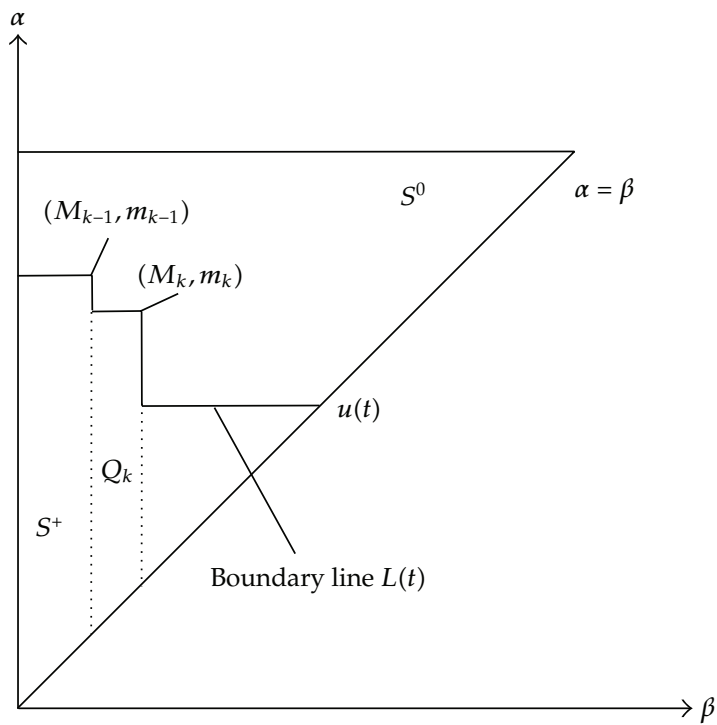

Figure 8: Numerical implementation of the Preisach model in the case of decreasing input.

Consequently, in the case of increasing input, as shown in Figure 7, the final link of boundary interface line $L(t)$ is horizontal line $M_{n}=u(t)$ and output can be determined as

$$
y(t)=\sum_{k=1}^{n(t)-1}\left[F\left(M_{k}, m_{k-1}\right)-F\left(M_{k}, m_{k}\right)\right]+F\left(u(t), m_{n-1}\right)
$$




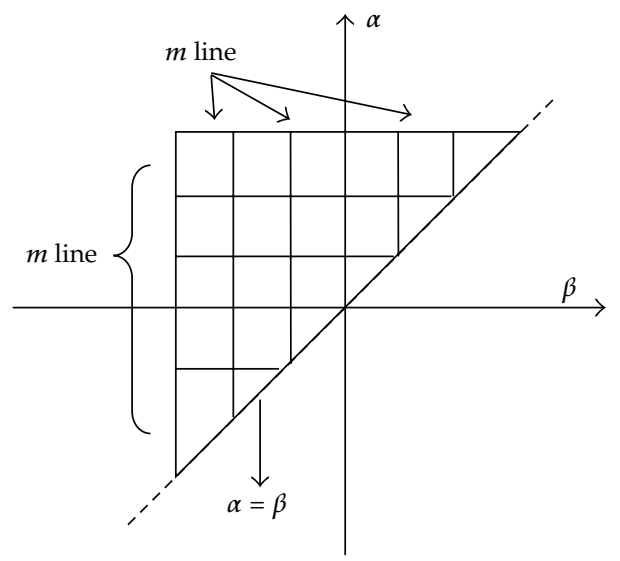

Figure 9: Discrete Preisach plane of $\Omega$.

Therefore, it can be obviously seen that the output of numerical Preisach model can be calculated from current input value, as well as minima and maxima history terms. The $F(\alpha, \beta)$ function in desired points of $\alpha$ and $\beta$ can be calculated by use of interpolation process on the batch of experimental data sample of hysteresis on the first-order reversal curves (ascending and descending). There are typical interpolation algorithms which can be used for this process such as cubic spline, nearest neighbor, linear interpolation, and Newton method.

\section{Numerical Preisach Implementation}

In order to implement the discussed numerical Preisach model, at first some experimental data is needed. By using the experimental data of the major ascending (or descending) loop and its attached first-order decreasing (or increasing) curves, a square mesh covering the Preisach plane $\Omega$ is obtained (see Figure 9). Any point $\left(\beta_{j}, \alpha_{i}\right)$ on the Preisach plane $\Omega$ can be considered as a vertex on a memory interface $L(t)$ which is formed by increasing input from the negative saturation state to a value $\alpha_{\mathrm{i}}$ and then decreasing input to a state as $\beta_{j}$. Different pairs of inputs $\left(\beta_{j}, \alpha_{i}\right)$ with $\alpha_{i} \geq \beta_{j}$ from different first-order reversal curves inside the major ascending curve divide the Preisach plane into small cells. In the preprocessing stage (training process) of the numerical Preisach model by dividing the Preisach plane $\Omega$ uniformly, using $m$ horizontal lines and $m$ vertical $l$ lines respectively, a total number of $N$ nodes on the Preisach plan $\Omega$ can be computed as

$$
N=\frac{(m+3)(m+2)}{3}
$$

In order to improve the prediction accuracy of the Preisach model, the equidistributed point should be large, and this greatly increases the complexity and cost of training process.

At the processing stage by entering an arbitrary input history and current values of input, the alternating series of dominant input extrema $\left\{M_{k}, m_{k}\right\}$ is first determined and for each new instant of time is updated. Finally, at the postprocessing stage (validation process), since most vertices of the memory interface $L(t)$ of the input do not coincide with those 

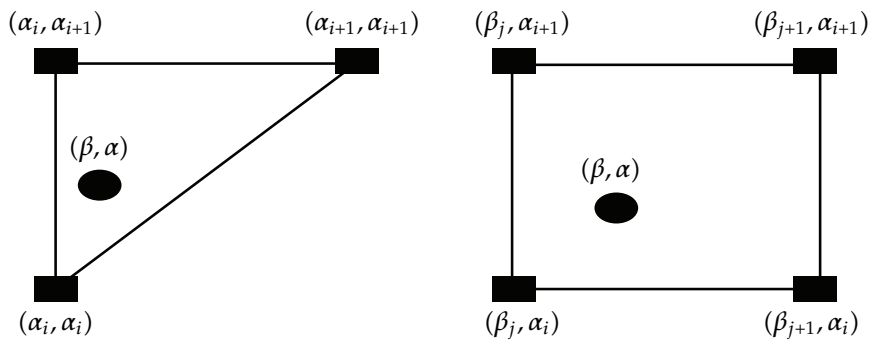

Figure 10: Calculation of $F(\alpha, \beta)$ in the postprocessing stage of numerical Preisach model by interpolation method.

discrete nodes of the discrete Preisach plane obtained in the preprocessing stage, the data of $F(\alpha, \beta)$ of these points should be obtained through interpolation [14].

This is done by first determining particular square or triangle cells to which points $\left(\mathrm{M}_{\mathrm{k}}, \mathrm{m}_{\mathrm{k}-1}\right),\left(\mathrm{M}_{\mathrm{k}}, \mathrm{m}_{\mathrm{k}}\right),\left(M_{n}, m_{n-1}\right),\left(M_{n}, u(t)\right)$, and $\left(u(t), m_{n-1}\right)$ belong. If a point $(\beta, \alpha)$ satisfies the condition $\left(\alpha-\alpha_{i}\right)\left(\alpha_{i}-\alpha_{i+1}\right) \leq 0$ and $\left(\beta-\beta_{j}\right)\left(\beta-\beta_{j+1}\right) \leq 0$, then this point is located inside a rectangular cell (see Figure 10) with vertices $\left(\beta_{j}, \alpha_{i}\right),\left(\beta_{j+1}, \alpha_{i}\right),\left(\beta_{j+1}, \alpha_{i+1}\right)$, and $\left(\beta_{j}, \alpha_{i+1}\right)$ (for which the data of $F(\alpha, \beta)$ in these points are already experimentally measured in the processing stage). On the other hand if the point $(\beta, \alpha)$ satisfies the condition $\left(\alpha-\alpha_{i}\right)\left(\alpha-\alpha_{i+1}\right) \leq$ 0 then the point $(\beta, \alpha)$ is located inside a triangle cell with vertices $\left(\alpha_{i}, \alpha_{i}\right),\left(\alpha_{i+1}, \alpha_{i+1}\right)$ and $\left(\alpha_{i}, \alpha_{i+1}\right)$. Next, the value of $F(\alpha, \beta)$ at those mentioned points can be computed by means of interpolation (cubic spline, nearest neighbor, linear interpolation, Newton method, etc.) of the mesh values of $F(\alpha, \beta)$ at the vertices of the above cells. Finally, the current values of output are evaluated by employing the formulae (3.9) and (3.10).

It is clear that in order to get the accurate results from the numerical Preisach model by the numerical interpolation methods, it is essential to have much data when the training process is performed. It means that the better Preisach plane $\Omega$ is divided in the training process (the larger $m$ in (4.1)) and the results are more accurate for calculation of $F(\alpha, \beta)$ from (3.9) and (3.10) through the interpolation in the validation process.

\section{Density Function Approximation}

In this section a numerical density function approximation method proposed by Shirley and Venkataraman [16] is presented. It is based on mapping Preisach model into a linear equation system and tries to solve this equation in order to find best fit solution.

Often because of inadequate number of experimental data, there are some limitations to predict $\mu(\alpha, \beta)$. Let us consider $\alpha-\beta$ plane as a partitioned plane; so (2.4) can be restated as

$$
f(t)=\sum_{i \in S_{+1}} \mu_{i}(\alpha, \beta)+\sum_{j \in S_{-1}}\left(-\mu_{j}(\alpha, \beta)\right) .
$$

In (5.1) it is supposed that a discrete density function with finite number of $\mu(\alpha, \beta)$ can be expressed by a vector and is denoted by $\psi(\alpha, \beta)$. Now, a state vector $\left(\widehat{S}_{i}\right)$ is defined, 
indicating state of each $\gamma_{\alpha, \beta}(-1$ or +1$)$, in accordance with each input-output experimental data; thus, (5.1) can be formulized as

$$
\begin{gathered}
f_{1}=\widehat{S}_{1}(\alpha, \beta) \cdot \psi(\alpha, \beta) \\
f_{2}=\widehat{S}_{2}(\alpha, \beta) \cdot \psi(\alpha, \beta) \\
\vdots \\
f_{m}=\widehat{S}_{m}(\alpha, \beta) \cdot \psi(\alpha, \beta) \\
\text { Let } F=\left[\begin{array}{c}
f_{1} \\
\vdots \\
f_{m}
\end{array}\right] \text { and } S=\left[\begin{array}{c}
\widehat{S}_{1} \\
\vdots \\
\hat{S}_{m}
\end{array}\right] ; \text { so }(5.1) \text { can be modeled as a linear equation problem }
\end{gathered}
$$

with unknown variable $\psi$ as

$$
F=S \times \psi(\alpha, \beta) .
$$

For $m$ input experimental data, it is necessary to find the best fit density function which satisfies desired output. When $m$ would be large enough, this linear equation system has no single solution. Therefore, the following equation should be minimized in order to reach this purpose:

$$
\varepsilon=\|S \psi-F\|^{2} \quad \psi>0
$$

where $\psi$ is target vector variable with $n$ element same as the number of finite $\gamma_{\alpha_{i} \beta_{j}}$. It should be mentioned that one limitation happens when $m$ is not comparable with $n$ (i.e., $\operatorname{rank}(S)<$ $\min \{m, n\})$. To overcome this problem, let $\operatorname{rank}(S)=q<\min (m, n)$. Then, by performing the singular decomposition on $S^{T} S$, there is $S^{T} S=Q A Q^{T}$, where $A$ is an $n \times n$ diagonal matrix and $\operatorname{rank}(A)=q<n$. After zero rows and zero columns elimination of matrix $A$ as well as removing the corresponding columns of $Q$, we have $\widetilde{A}$ and $\widetilde{Q}$ for which $\widetilde{Q} \widetilde{A} \widetilde{Q}^{T}=S^{T} S$; thus (5.3) and (5.4) can be modified as the following equation which should be minimized in order to find $Z$ and then $\psi$ :

$$
\varepsilon=Z^{T} \tilde{A} Z-F^{T} S \widetilde{Q} Z \quad \psi=\widetilde{Q} Z .
$$

In the sequel, the density function can be approximately calculated for a set of experimental data. The main advantage of Shirley method is its good ability of hysteresis systems identification without definition any of pre-defined density function. But, it is still not convenient enough in control applications especially in real-time control. It comes back to the fact that solving the above optimization problem is so time-consuming process.

\section{Preisach Neural Network Approach}

Regarding (3.9) and (3.10), it is illustrated that in numerical Preisach modeling, output value depends on local minima and local maxima, of input history by $F(\alpha, \beta)$ functionality. 


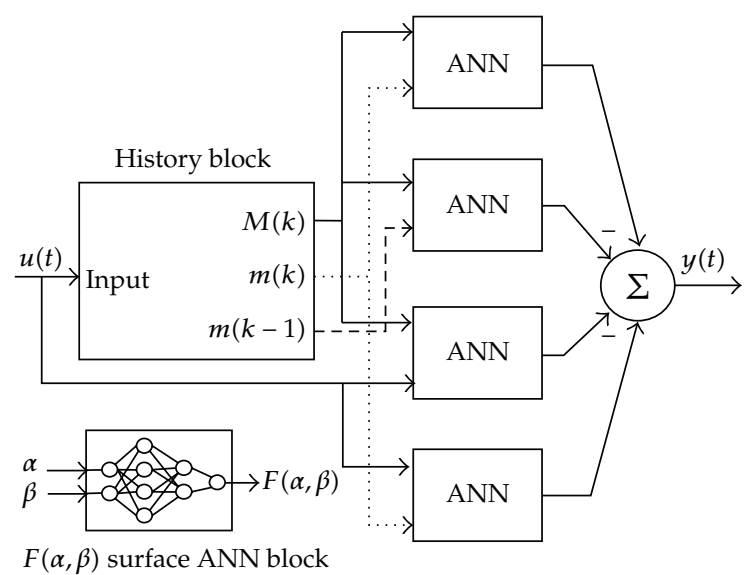

Figure 11: The proposed ANN-based Preisach model.

Consequently, this model needs a memory block which saves $M_{k}$, local maxima, and $m_{k}$, local minima of input at previous instants of time. $F(\alpha, \beta)$ is a suppositional surface on $\alpha-\beta$ plane which depends on hysteresis nature and can be defined numerically by experimental data of real system [25].

ANN is a computing system made up of a number of simple, highly interconnected processing elements, neurons, which processes information in parallel by its dynamic state response to external inputs [27]. Also ANN has powerful fault tolerant computing ability which has been used to model a wide range of systems for which mathematical models either cannot be defined or are ill-defined. Similarly, ANNs are well suited for systems that involve complex, multivariable processes with time variant parameters. As a result, ANN seems to be a good and powerful tool for approximation of $F(\alpha, \beta)$ surface.

In Figure 11, the proposed ANN-based numerical Preisach model is shown. In this method surface of $F(\alpha, \beta)$ is realized by two-dimensional input and single output feedforward multilayer Perceptron neural network structure. It has two hidden layers with 15 and 5 neurons with tangent sigmoid activation function, while the activation function of output layer neurons is linear. Also, learning algorithm is Back-Propagate (BP). BP is based on error correction learning rule and can be considered as an extension of the mean least squares algorithm. This learning algorithm is used to adjust the network weights and biases in order to minimize the output error of the network [28]. The process of presenting batches of training cases to the network continues till the average error over the entire training set reaches a defined error goal or any other convergence criterion is achieved.

The history block works like a decision box and prepares $M_{k}$ and $m_{k}$ values for ANN base processing layer. In other words, it compares current input with the last input and updates maxima and minima values. It is considerable that history block should have wipingout property. It means that each local maximum wipes out the vertices whose $\alpha$ coordinates are below this maximum, and each local minimum wipes out the vertices whose $\beta$ coordinates are above this minimum. It is equivalent to the erasing of the history associated with these vertices. Thus, subsequent variations of input might erase some previous history [25].

In the next section, evaluation of presented approach is compared with the numerical classical Preisach method as well as the discussed Shirley method, precisely. 


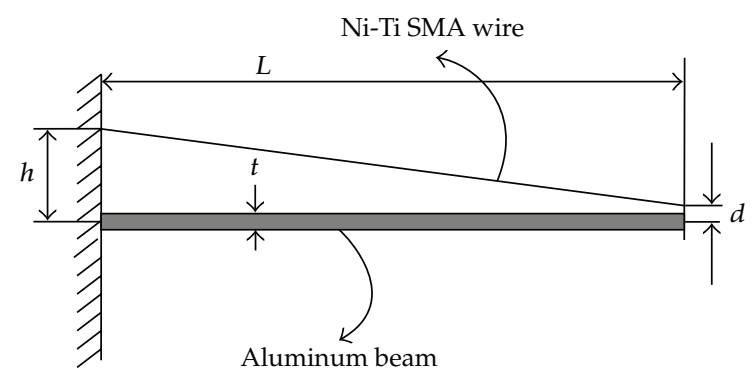

Figure 12: General structure of experimental apparatus.

Table 1: Parameters of experimental apparatus.

\begin{tabular}{lcccccc}
\hline Parameter & $\mathrm{l}$ & $\mathrm{t}$ & $\mathrm{d}$ & $\mathrm{E}$ & $\mathrm{b}$ (beam width) & $\mathrm{h}$ \\
\hline Value & $400 \mathrm{~mm}$ & $1.27 \mathrm{~mm}$ & $100 \mathrm{~mm}$ & $70 \mathrm{GPa}$ & $25 \mathrm{~mm}$ & $100 \mathrm{~mm}$ \\
\hline
\end{tabular}

\section{Experimental Results}

Today there is wide range of SMA commercial applicability in many devices such as microrobots, medical and dental tools, nonexplosive aerospace actuators, and pneumatic microvalve [29]. In addition, recently SMA wires are being used as an intelligent material in new generation smart structures, which the shape of the structure would be controllable by use of SMA actuators. However, due to the deflection and displacement hysteresis behavior in SMA and Piezo actuators, there is rigorous hysteresis nonlinearity in these actuators which make their modeling and control more difficult. Consequently, accurate identification of these actuators behavior is one of the interesting and challenging fields in automation and control.

For evaluation of the proposed ANN-based Preisach hysteresis model, numerical classical Preisach model, and Shirley approach, a one-dimensional flexible aluminum beam whose deflection is controlled by an SMA wire as an actuator is used. This SMA actuator is made of Nitinol (Ni-Ti) alloy which has excellent electrical and mechanical properties, long fatigue life, and high corrosion resistance, and due to these properties this material is used in many SMA actuators today [30].

General structure of the experimental apparatus is shown in Figure 12. In this experimental setup a Flexinol TM actuator wire, manufactured by Dynalloy Inc, is used. This NiTi SMA actuator wire is a one-way high-temperature $\left(90^{\circ} \mathrm{C}\right)$ shape memory with 0.01 -inch diameter. Parameters of the experimental apparatus and SMA wire according to Figure 12 are presented in Tables 1 and 2, respectively. The beam end deflection was measured by a highprecision linear potentiometer and fed to the computer through a multifunction (A/D, D/A) Advantech PCI-1711 card. The actuation input of the experimental apparatus is the current applied to the SMA wire and obtained from a D/A card and a V/I converter.

Since the input of the hysteresis model is assumed to be the temperature of the SMA wire, it is essential to determine the temperature of the wire. The temperature of the SMA wire is measured by two very thin $(0.02 \mathrm{~mm}$ probe diameter) $J$ thermocouples attached by a very conductive paste to both ends of the SMA wire. The average value obtained by the thermocouples is selected as the SMA wire temperature and fed to the computer through the mentioned multifunction card. This system is controlled in real time with real-time Windows Target Toolbox of MATLAB. 
Table 2: Physical parameters of SMA wire actuator.

\begin{tabular}{lc}
\hline Parameter & Value \\
\hline$M_{\mathrm{f}}$ & $43.9^{\circ} \mathrm{C}$ \\
$M_{\mathrm{s}}$ & $48.4^{\circ} \mathrm{C}$ \\
$A_{\mathrm{s}}$ & $68^{\circ} \mathrm{C}$ \\
$A_{\mathrm{f}}$ & $73.75^{\circ} \mathrm{C}$ \\
$C_{\mathrm{A}}$ & $6.73 \mathrm{MPa} /{ }^{\circ} \mathrm{C}$ \\
$C_{\mathrm{M}}$ & $6.32 \mathrm{MPa} /{ }^{\circ} \mathrm{C}$ \\
$\varepsilon_{L}$ & $4.10 \%$ \\
$E_{\mathrm{A}}$ & $31.5 \mathrm{Gpa}$ \\
$E_{\mathrm{M}}$ & $20 \mathrm{Gpa}$ \\
$\sigma_{S}$ & $25 \mathrm{MPa}$ \\
$\sigma_{f}$ & $78 \mathrm{MPa}$ \\
\hline
\end{tabular}

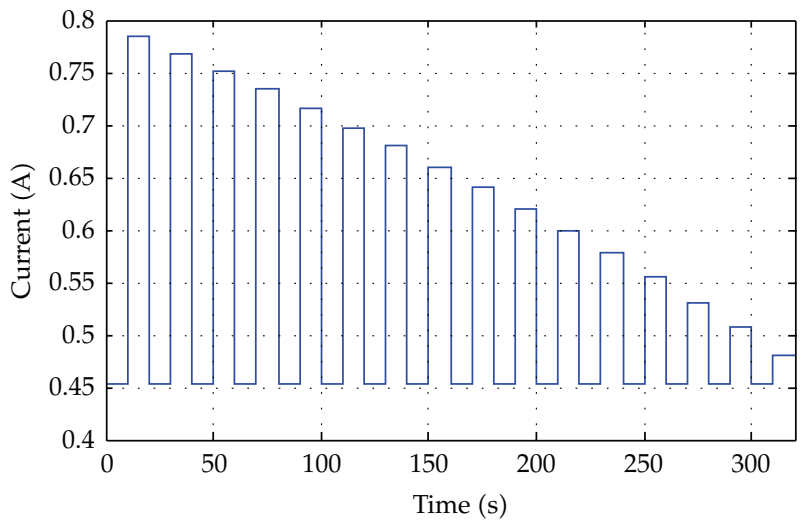

Figure 13: The decaying step current applied in the training process.

The input current applied to the SMA actuator in the training process is a decaying step signal and is shown in Figure 13. The corresponding beam deflection SMA wire temperature profile, obtained due to the applied current, is also shown in Figure 14. In the training process of the mentioned models 153 data set, consisting of the major loop and 15 firstorder descending reversal curves, is used in order to approximate $F(\alpha, \beta)$ surface. For each switching point $(\alpha, \beta)$, according to (3.4), the corresponding $F(\alpha, \beta)$ is computed by measuring the output beam end deflection as the input temperature is increased to $\alpha$ and then decreased to $\beta$. Likewise, in Figure 15 surface of $F(\alpha, \beta)$ function which has been realized by ANN is also presented. Also to identify hysteresis system by using Shirley approach, density function should be approximated too. In the five sections, we described how this method can realize Preisach model by density function approximation based on finding best fit solution of a linear equation system. For hysteresis system modeling by Shirley approach, the same data set was used with 80 partitions for $\alpha-\beta$ plane [16]. The identified density function by this method is presented in Figure 16.

For evaluation of the prediction of the output beam end deflection by the numerical classical Preisach model, the proposed ANN-based Preisach model, and the Shirley approach, with respect to the experimental data, in the first validation process the current profile shown in Figure 17 is applied to the SMA actuator. In Figure 18 the prediction of the hysteresis 


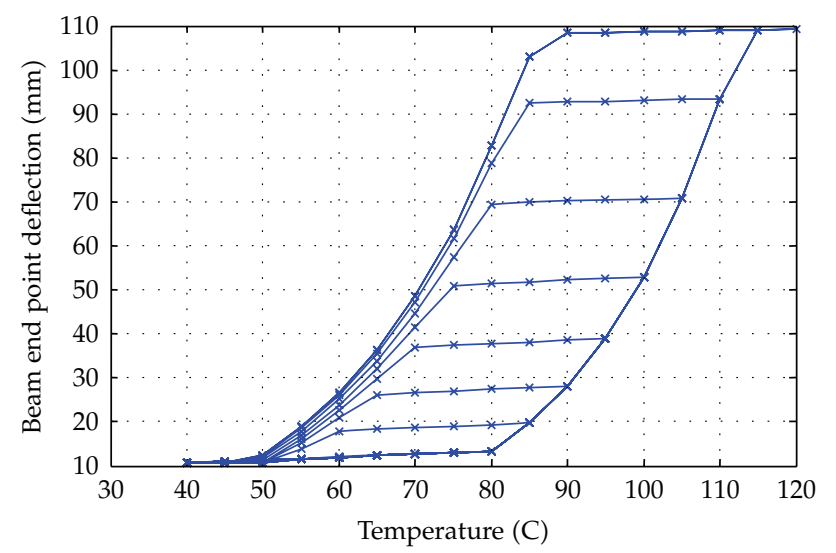

Figure 14: Hysteresis behavior between the beam end point deflection and the SMA wire temperature in the training process.

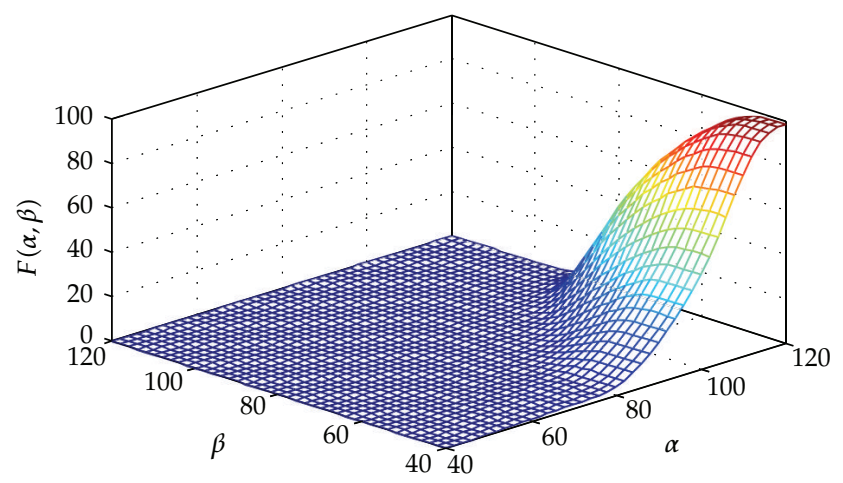

Figure 15: Surface $F(\alpha, \beta)$ identified by ANN using training data set.

behavior between the beam end point deflection and the SMA temperature by the Preisach model, the Shirley method as well as the proposed ANN method is compared by the experimental data. As it is seen from this figure none of the first-order transition curves applied in this validation process is applied in the training process. As it is clear in Figure 18 the proposed ANN based Preisach model can identify hysteresis in first order reversal curves more accurately than both the classical numerical Preisach model and the Shirley method. In order to show this property more clearly, the absolute error of the three mentioned models with respect to the experimental data is shown in Figure 19. The maximum, mean, and mean square values of absolute error for the three methods are also presented in Table 3.

For better evaluation of the three mentioned methods in predicting the hysteresis behavior of higher-order minor loops, in the second validation process the damped current profile shown in Figure 20 is applied to the SMA actuator. The prediction of the hysteresis behavior of higher-order minor loops by the Preisach model, Shirley approach, and the proposed ANN method is compared by the experimental data in Figure 21. This figure demonstrates the power ability of the proposed ANN model, with respect to two other models, in higher order reversal curves prediction. Indeed, it comes back to the general approximation capability of ANNs. Also, Figure 22 shows the absolute error of the three considered models with respect to the experimental data. In addition, the maximum, mean, and mean square values of absolute error for the three methods are presented in Table 4 . 


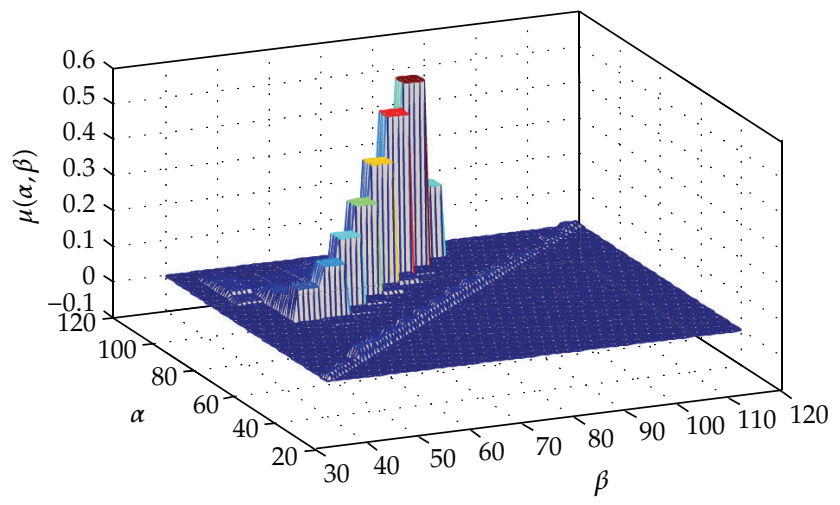

Figure 16: Approximated $\mu(\alpha, \beta)$ surface by use of linear equation system optimization (Shirley Approach).

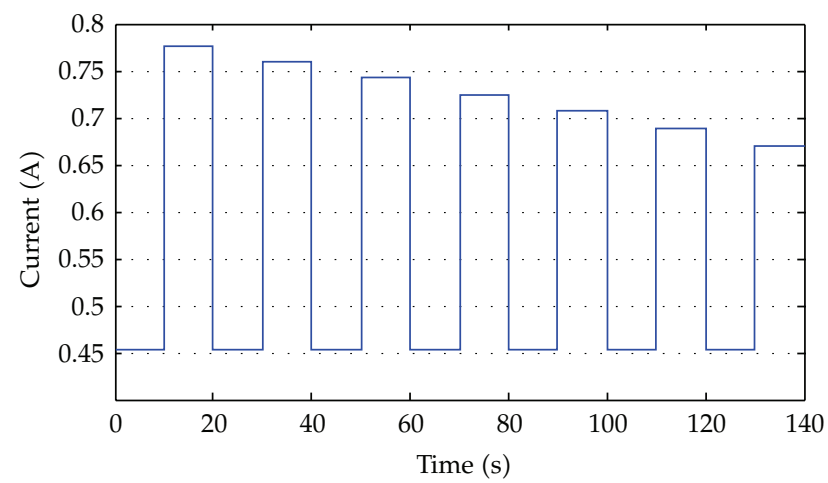

Figure 17: The decaying step current applied in the first validation process.

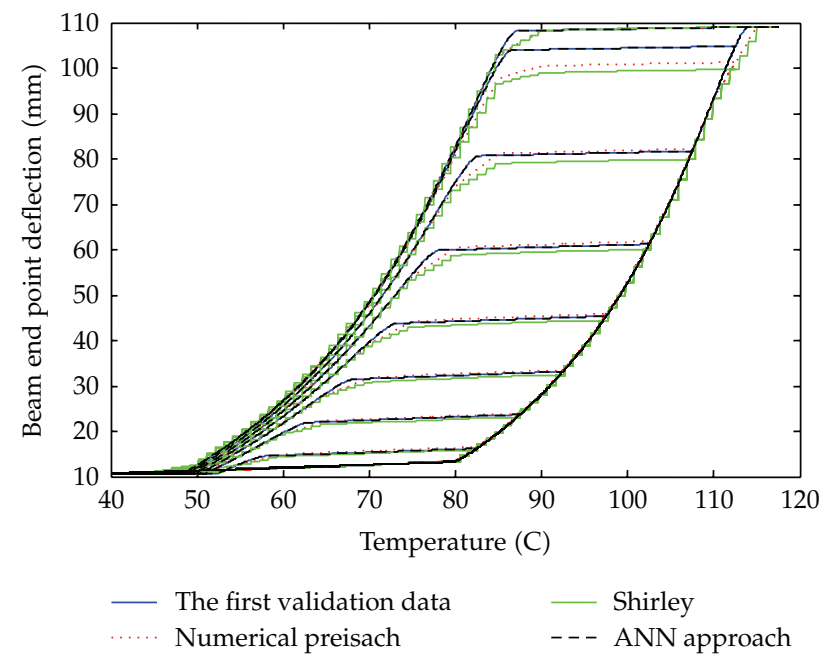

Figure 18: Prediction of the hysteresis behavior for the classical numerical Preisach, Proposed ANN Model and Shirley Approach in comparison to the first experimental data set. 


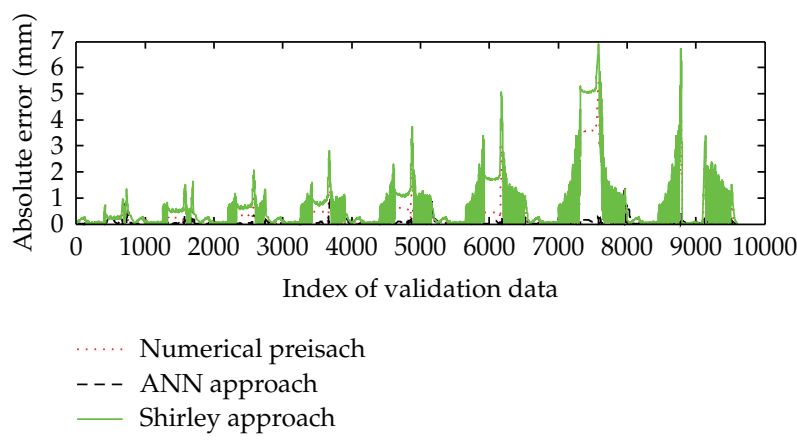

Figure 19: Absolute error of the Classical Numerical Preisach model, the Proposed ANN Model and the Shirley Method in comparison with the first experimental data set.

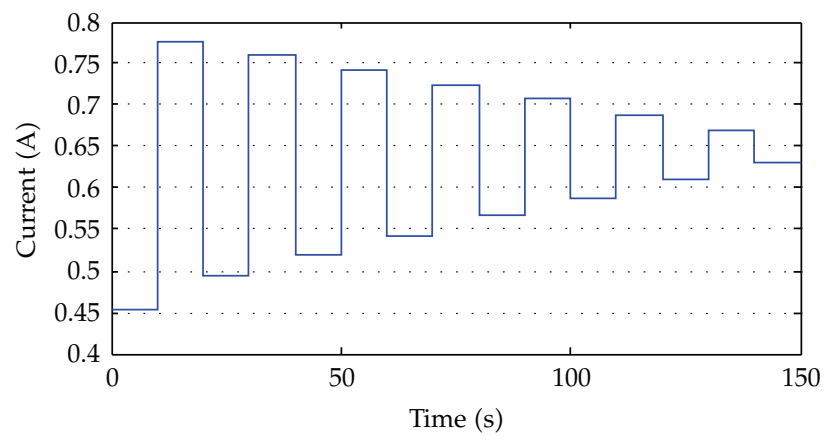

Figure 20: The decaying step current applied in the second validation process.

Table 3: Error of the three considered models in the first validation process.

\begin{tabular}{lccc}
\hline & $\begin{array}{c}\text { Mean absolute error } \\
(\mathrm{mm})\end{array}$ & $\begin{array}{c}\text { Max of absolute error } \\
(\mathrm{mm})\end{array}$ & $\begin{array}{c}\text { Mean square of } \\
\text { absolute error } \\
(\mathrm{mm})\end{array}$ \\
\hline $\begin{array}{l}\text { Numerical Preisach } \\
\text { model }\end{array}$ & 0.3228 & 5.4439 & 0.6145 \\
Shirley method & 0.644 & 6.907 & 1.505 \\
Proposed ANN model & 0.1315 & 1.36 & 0.25 \\
\hline
\end{tabular}

Table 4: Error of the three considered models in the second validation process.

\begin{tabular}{lccc}
\hline & $\begin{array}{c}\text { Mean absolute error } \\
(\mathrm{mm})\end{array}$ & $\begin{array}{c}\text { Max of absolute error } \\
(\mathrm{mm})\end{array}$ & $\begin{array}{c}\text { Mean square of } \\
\text { absolute error } \\
(\mathrm{mm})\end{array}$ \\
\hline $\begin{array}{l}\text { Numerical Preisach } \\
\text { model }\end{array}$ & 1.5147 & 5.3964 & 4.2677 \\
Shirley Method & 1.78 & 6.827 & 5.629 \\
Proposed ANN model & 0.46 & 3.533 & 0.549 \\
\hline
\end{tabular}




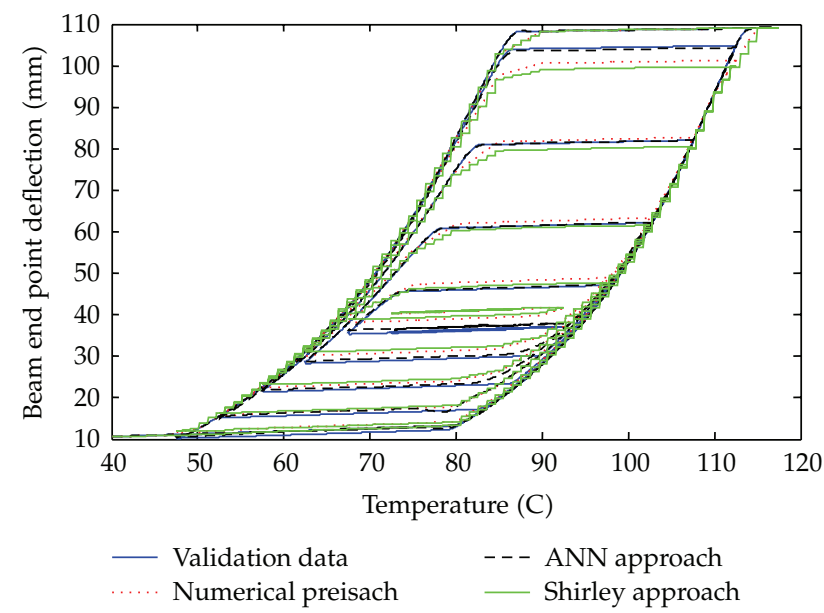

Figure 21: Prediction of the hysteresis behavior for classical numerical Preisach model, proposed ANN model, and Shirley approach in comparison to the second damped experimental data set.

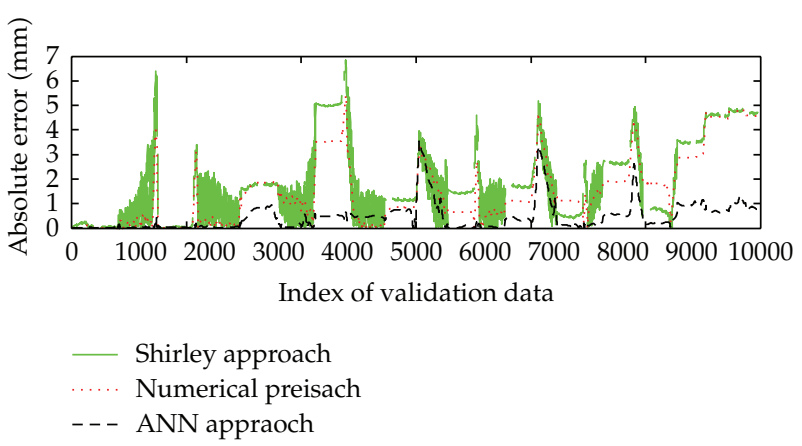

Figure 22: Absolute error of the classical numerical Preisach Model, the proposed ANN model, and the Shirley method in comparison with the second damped experimental data set.

As it is concluded from Figures 19 and 22 and Tables 3 and 4, the proposed ANN model has more accurate predictions than the numerical Preisach and Shirley models. In all of these validation processes the mean error of numerical Preisach model is better than the Shirley method while it is triple of corresponding error in the proposed ANN model. It is demonstrated (but not shown in this paper) that in order to bring the mean square error of the Preisach model (in the second validation process) at the order of the corresponding value of ANN model, the training should be done with 420 data instead of 153 data that was used first. It means that in order to have precise results in the numerical Preisach model, much data should necessarily be used, and this directly increases the experimental cost of training process. The significant decrease in the error of the proposed ANN model seems more valuable when it is known that in this ANN model, unlike the fuzzy inference engines, there is a straightforward training algorithm which enables utilizing this approach for many other hysteresis systems without any change in the structure of the ANN model. 


\section{Conclusion}

In this paper, a novel hysteresis identification method based on numerical classical Preisach model by use of artificial neural networks (ANNs) has been presented. Since the accuracy of the Preisach function approximation methods is strongly dependent on the type of the candidate Preisach function and the number of its parameters, this approach remedies these drawbacks. In addition, this approach does not suffer from a lack of learning algorithms to adjust the system parameters existed in fuzzy inference engine.

The experimental data showed that this ANN model can predict SMA actuators hysteresis behavior with considerable accuracy in comparison with numerical Preisach model. It also has powerful ability to precisely predict the higher-order hysteresis minor loops behavior even though it is only trained by first-order reversal data. Therefore, it is a convenient method for many applications such as hysteresis nonlinearity control, hysteresis identification, and realization for performance evaluation in some physical systems such as magnetic and SMA materials.

\section{References}

[1] B. K. Nguyen and K. K. Ahn, "Feedforward control of shape memory alloy actuators using fuzzybased inverse Preisach model," IEEE Transactions on Control Systems Technology, vol. 17, no. 2, pp. 434-441, 2009.

[2] X. Tan and R. V. Iyer, "Modeling and control of hysteresis," IEEE Control Systems Magazine, vol. 29, no. 1, pp. 26-28, 2009.

[3] V. Basso, C. P. Sasso, and M. LoBue, "Thermodynamic aspects of first-order phase transformations with hysteresis in magnetic materials," Journal of Magnetism and Magnetic Materials, vol. 316, no. 2, pp. 262-268, 2007.

[4] S. Cao, B. Wang, R. Yan, W. Huang, and Q. Yang, "Optimization of hysteresis parameters for the JilesAtherton model using a genetic algorithm," IEEE Transactions on Applied Superconductivity, vol. 14, no. 2, pp. 1157-1160, 2004.

[5] J. V. Leite, S. L. Avila, N. J. Batistela et al., "Real coded genetic algorithm for Jiles-Atherton model parameters identification," IEEE Transactions on Magnetics, vol. 40, no. 2, pp. 888-891, 2004.

[6] K. K. Ahn and N. B. Kha, "Modeling and control of shape memory alloy actutors using Presiach model, genetic algorithm and fuzzy logic," Journal of Mechanical Science and Technology, vol. 20, no. 5, pp. 634-642, 2008.

[7] R. B. Gorbet, Control of Hysteresis Systems with Preisach Represtations, Ph.D. thesis, University of Waterloo, Ontario, Canada, 1997.

[8] S. Mittal and C. H. Menq, "Hysteresis compensation in electromagnetic actuators through Preisach model inversion," IEEE/ASME Transactions on Mechatronics, vol. 5, no. 4, pp. 394-409, 2000.

[9] X. Tan and J. S. Baras, "Modeling and control of hysteresis in magnetostrictive actuators," Automatica, vol. 40, no. 9, pp. 1469-1480, 2004.

[10] D. Hughes and J. T. Wen, "Preisach modeling of piezoceramic and shape memory alloy hysteresis," Smart Materials and Structures, vol. 6, no. 3, pp. 287-300, 1997.

[11] S. R. Viswamurthy and R. Ganguli, "Modeling and compensation of piezoceramic actuator hysteresis for helicopter vibration control," Sensors and Actuators, A: Physical, vol. 135, no. 2, pp. 801-810, 2007.

[12] K. K. Ahn and N. B. Kha, "Improvement of the performance of hysteresis compensation in SMA actuators by using inverse Preisach model in closed-loop control system," Journal of Mechanical Science and Technology, vol. 20, no. 5, pp. 634-642, 2006.

[13] K. K. Ahn and N. B. Kha, "Internal model control for shape memory alloy actuators using fuzzy based Preisach model," Sensors and Actuators, A: Physical, vol. 136, no. 2, pp. 730-741, 2007.

[14] I. D. Mayergoyz, Mathematical Models of Hysteresis and their Applications, Elsevier Science, New York, NY, USA, 2003.

[15] A. A. Adly and S. K. Abd-El-Hafiz, "Using neural networks in the identification of preisach-type hysteresis models," IEEE Transactions on Magnetics, vol. 34, no. 3, pp. 629-635, 1998. 
[16] M. E. Shirley and R. Venkataraman, "On the Identification of Preisach Measures," in Smart Structures and Materials 2003 Modeling, Signal Processing, and Control, vol. 5049 of Proceedings of SPIE, pp. 326-336, September 2003.

[17] M. Cirrincione, R. Miceli, G. R. Galluzzo, and M. Trapanese, "A novel neural approach to the determination of the distribution function in magnetic Preisach systems," IEEE Transactions on Magnetics, vol. 40, no. 4, pp. 2131-2133, 2004.

[18] C. Natale, F. Velardi, and C. Visone, "Identification and compensation of Preisach hysteresis models for magnetostrictive actuators," Physica B, vol. 306, no. 1-4, pp. 161-165, 2001.

[19] E. Dlala and A. Arkkio, "A neuro-fuzzy-based Preisach approach on hysteresis modeling," Physica B, vol. 372, no. 1-2, pp. 49-52, 2006.

[20] J. S. R. Jang, "ANFIS: adaptive-network-based fuzzy inference system," IEEE Transactions on Systems, Man and Cybernetics, vol. 23, no. 3, pp. 665-685, 1993.

[21] J. DA. Wei and C. T. Sun, "Constructing hysteretic memory in neural networks," IEEE Transactions on Systems, Man, and Cybernetics, Part B: Cybernetics, vol. 30, no. 4, pp. 601-609, 2000.

[22] E. Kolman and M. Margaliot, Knowledge-Based Neurocomputing, Springer, Berlin, Germany, 2009, STUDFUZZ 234

[23] F. Preisach, “Über die magnetische Nachwirkung,” Zeitschrift für Physik, vol. 94, no. 5-6, pp. 277-302, 1935.

[24] M. A. Krasnoselskii and A. V. Pokrovskii, Systems with Hysteresis, Springer, Berlin, Germany, 1983.

[25] I. D. Mayergoyz, "Mathematical models of hysteresis," IEEE Transactions on Magnetics, vol. 22, no. 5, pp. 603-608, 1986.

[26] Z. Bo and D. C. Lagoudas, "Thermomechanical modeling of polycrystalline SMAs under cyclic loading, Part IV: modeling of minor hysteresis loops," International Journal of Engineering Science, vol. 37, no. 9, pp. 1205-1249, 1999.

[27] R. Hecht-Nielsen, Neurocomputing, Addison-Wesley, Reading, Mass, USA, 1989.

[28] D. E. Rumelhart, G. E. Hinton, and R. J. Williams, Learning Internal Representations by Error Propagation, Parallel Data Processing, vol. 1, MIT Press, Cambridge, Mass, USA, 1986.

[29] C. M. Wayman, T. W. Duerig et al., Engineering Aspects of Shape Memory Alloys, ButterworthHeinemann, Oxford, UK, 1990.

[30] M. Novotny and J. Kilpi, "Shape Memory Alloys (SMA)," http://www.ac.tut.fi/aci/courses / ACI51106/pdf/SMA/SMA-introduction.pdf. 


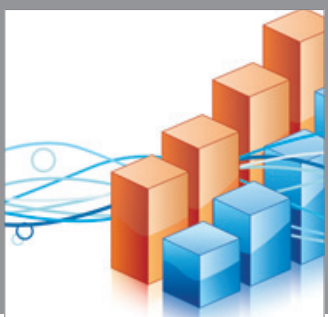

Advances in

Operations Research

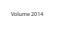

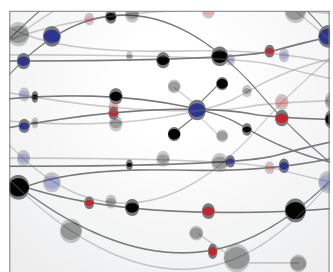

\section{The Scientific} World Journal
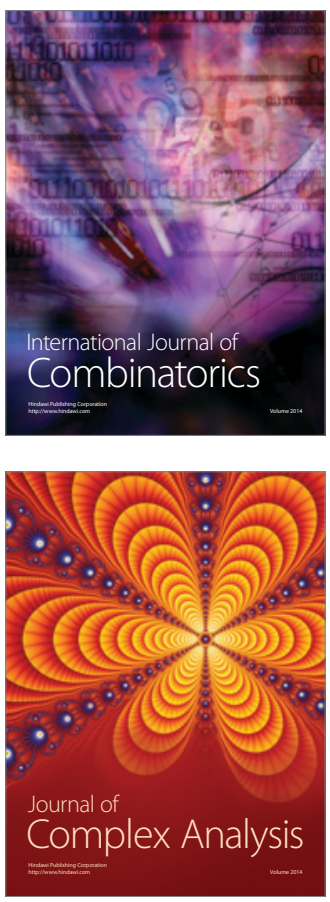

International Journal of

Mathematics and

Mathematical

Sciences
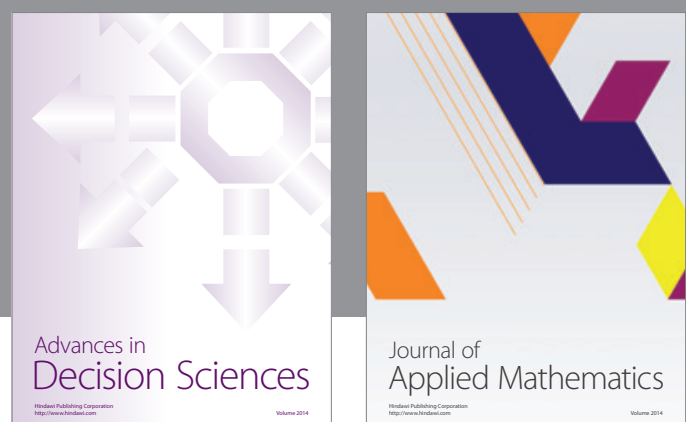

Journal of

Applied Mathematics
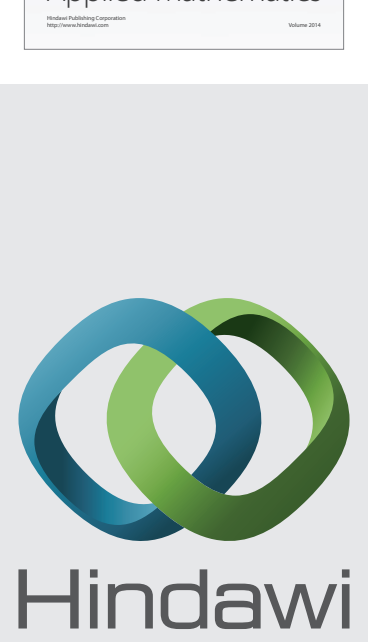

Submit your manuscripts at http://www.hindawi.com
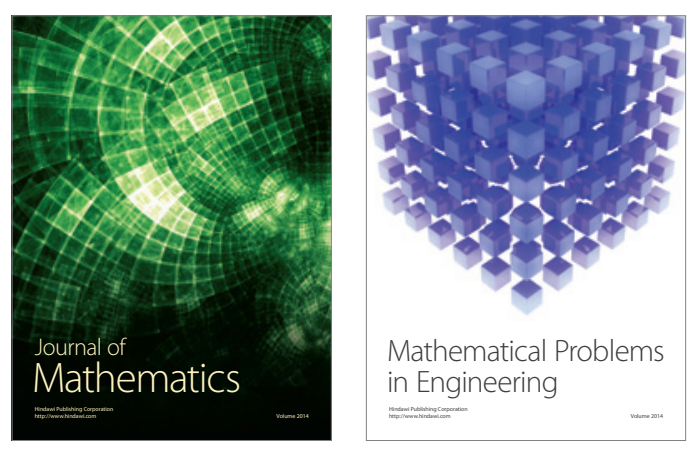

Mathematical Problems in Engineering
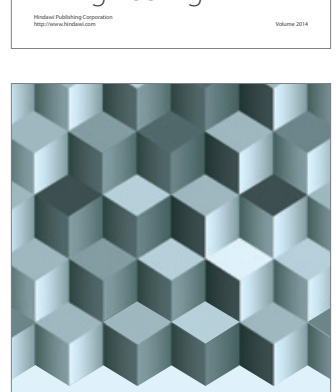

Journal of

Function Spaces
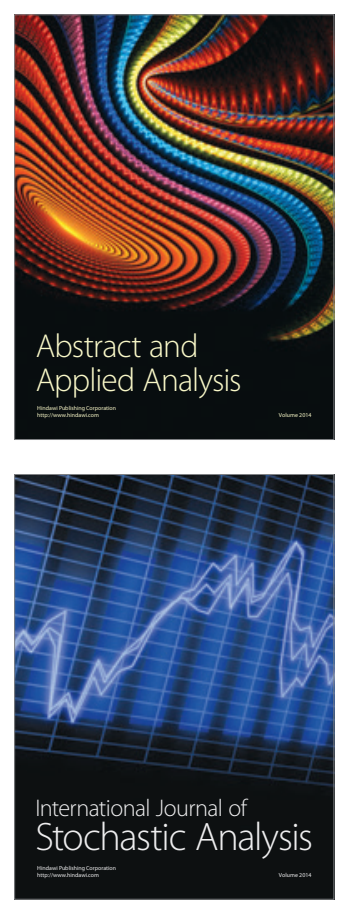

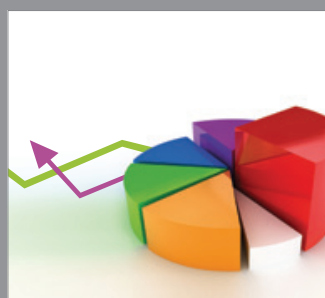

ournal of

Probability and Statistics

Promensencen
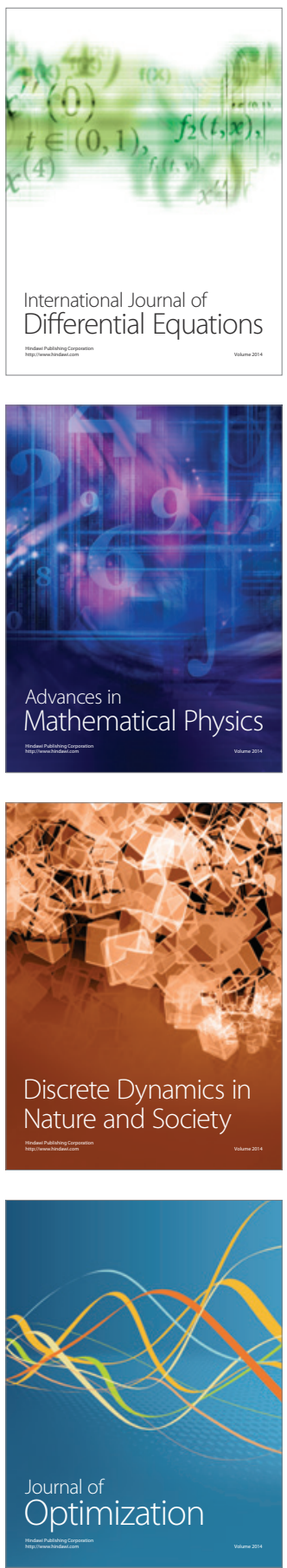